NASA/TM-1998-208407

\title{
Insights Into the Fractional Order Initial Value Problem via Semi-infinite Systems
}

Tom T. Hartley

University of Akron, Akron, Ohio

Carl F. Lorenzo

Lewis Research Center, Cleveland, Ohio

National Aeronautics and

Space Administration

Lewis Research Center 
Available from

NASA Center for Aerospace Information

7121 Standard Drive

Hanover, MD 21076

Price Code: A03
National Technical Information Service 5285 Port Royal Road Springfield, VA 22100 Price Code: A03 


\title{
Insights Into the Fractional Order Initial Value Problem Via Semi-Infinite Systems
}

\author{
Tom T. Hartley \\ Department of Electrical Engineering \\ The University of Akron \\ Akron, OH 44325-3904 \\ Carl F. Lorenzo \\ NASA Lewis Research Center \\ 21000 Brookpark Rd. \\ Cleveland, $\mathrm{OH} 44135$
}

\begin{abstract}
This paper considers various aspects of the initial value problem for fractional order differential equations. The main contribution of this paper is to use the solutions to known spatially distributed systems to demonstrate that fractional differintegral operators require an initial condition term that is time-varying due to past distributed storage of information.
\end{abstract}

\section{Introduction}

The problem to be considered here is the initial condition problem for systems with components of fractional mathematical order. Specifically, the case when the fractional components are of mathematical order $1 / 2$ is considered at length. This is due to the availability of a convenient physical system to use as reference, namely, the semi-infinite lossy line. Laplace transform techniques are utilized throughout the study, as they are the fundamental tool of linear system theory. The main contribution of this work is the demonstration that fractional differintegral operators require the addition of time-dependent initial condition terms in their application. The implication is that the solution of fractional ordinary differential equations will require that the problem statement include the appropriate time-dependent initial condition terms along with some information about the structure of these terms. It is also shown, by using a semi-infinite elastic bar, that the simple derivative or integral can require these terms as well. The present section provides some background into fractional order systems before the distributed line is addressed in the later sections.

The idea of fractional integrals and derivatives has been known since the development of the regular calculus, with the first reference probably being associated with Leibniz in 1695 [Oldham and Spanier (1974), page 3]. Although not well known to most engineers, the fractional calculus has been considered by prominent mathematicians [Courant and Hilbert (1953)] as well as the "engineers" of the operational calculus [Heaviside (1893)], [Bush (1929)]. In fact, many textbooks written before 1960 have a small section on fractional calculus [Goldman (1949)], [Holbrook (1966)], [Starkey (1954)], [Carslaw and Jeager (1941)], [Scott (1955)], [Mikusinski (1959)]. An outstanding historical survey can be found in Oldham and Spanier who also give what is unquestionably the most readable and complete mathematical presentation of the fractional 
calculus. Other bound discussions of the area are given by Ross, McBride, and McBride and Roach. Unfortunately, many of the results in the fractional calculus are given in the language of the mathematician and are not readily accessible to the general engineering community.

Many systems are known to display fractional order dynamics. Probably the first physical system to be widely recognized as demonstrating fractional behavior is the semi-infinite lossy (RC) line. Looking into the line, the operational impedance is

$$
\mathrm{Z}(\mathrm{s})=\frac{\mathrm{V}(\mathrm{s})}{\mathrm{I}(\mathrm{s})}=\frac{1}{\sqrt{\mathrm{s}}} .
$$

The current is thus equal to the half-derivative of the applied voltage (assuming time begins at $t=0$ ), which can be written in the time domain as,

$$
\mathrm{i}(\mathrm{t})=\frac{\mathrm{d}^{1 / 2} \mathrm{v}(\mathrm{t})}{\mathrm{dt}^{1 / 2}}
$$

Although studied by many, Heaviside considered this system extensively by using the operational calculus. He also states that "there is a universe of mathematics lying in between the complete differentiations and integrations" [Heaviside (1893), p. 459] This specific system is studied further in the next section. An equivalent system is the diffusion of heat into a semi-infinite solid. Here the temperature at the boundary is equal to the half integral of the heat rate there. Other systems that are known to display fractional order dynamics are viscoelastic systems [Bagley and Calico (1991)], [Koeller (1984)], [Koeller (1986)], [Skaar, Michel, and Miller (1988)], [LopezMarcos (1990)]; colored noise [Mandelbrot (1967)]; electrode-electrolyte polarization [Ichise, Nagayanagi, and Kojima (1971)], [Sun, Onaral, and Tsao (1984)]; dielectric polarization [Sun, Abdelwahab, and Onaral (1984)]; boundary layer effects in ducts [Sugimoto (1991)]; and electromagnetic waves [Heaviside (1893)]. As many of these systems depend upon specific material and chemical properties, it is expected that a wide range of fractional order behaviors are possible by considering different materials.

The two most common definitions for the general fractional differintegral are probably the Grünwald definition and the Riemann-Liouville definition [Oldham and Spanier (1974)]. The Riemann-Liouville definition is given here:

$$
\frac{d^{q} f(t)}{[d(t-a)]^{q}}=\frac{1}{\Gamma(-q)} \int_{a}^{t} \frac{f(\tau)}{(t-\tau)^{q+1}} d \tau, q<0
$$

where (a) represents the beginning of the domain of the differintegral. Here $q$ can have non-integer values, and thus the name fractional differintegral. Notice that the definition is based on integration, and more importantly is a convergent convolution integral for $q<0$. For fractional differentiation ( $q>0$ ), the usual integer $n^{\text {th }}$ derivative is take 1 of the fractional $(q-n)^{\text {th }}$ integral,

$$
\frac{d^{q} f}{d t^{q}}=\frac{d^{n}}{d t^{n}}\left[\frac{d^{q-n_{f}}}{d t^{q-n}}\right], \quad q>0 \text { and } n \text { an integer }>q
$$

The basic engineering tool for analyzing linear systems $i:$ the Laplace transform. The Laplace transform of Equation (1) as given by Oldham and Spanier vith $a=0$ is 


$$
L\left\{\frac{\mathrm{d}^{\mathrm{q}} \mathrm{f}(\mathrm{t})}{\mathrm{dt}^{\mathrm{q}}}\right\}=\mathrm{s} \mathrm{q} L\{\mathrm{f}(\mathrm{t})\}-\sum_{\mathrm{k}=0}^{\mathrm{n}-1} \mathrm{~s}^{\mathrm{k}}\left[\frac{\mathrm{d}^{\mathrm{q}-1-\mathrm{k}_{\mathrm{f}}(\mathrm{t})}}{\mathrm{dt}^{\mathrm{q}-1-\mathrm{k}}}\right]_{\mathrm{t}=0}, \text { for all } \mathrm{q},
$$

where $\mathrm{n}$ is an integer such that $\mathrm{n}-1<\mathrm{q}<\mathrm{n}$. The problem with this formulation is that the initial condition terms are fractional integrals and derivatives evaluated at time zero. Unfortunately, this information is not readily specified in many problems. Notice that when the initial conditions are assumed to be zero, this equation reduces to

$$
L\left\{\frac{\mathrm{d}^{\mathrm{q}} \mathrm{f}(\mathrm{t})}{\mathrm{dt}^{\mathrm{q}}}\right\}=\mathrm{s}^{\mathrm{q}} L\{\mathrm{f}(\mathrm{t})\} .
$$

It is the nature of the initial condition terms that motivates this study. The next section addresses this problem through a study of the semi-infinite lossy line.

\section{The Semi-Infinite Lossy Line}

This section contains a classical iterated Laplace transform derivation of the operational impedance for a semi-infinite lossy line. Of particular importance is the inclusion of the spatial initial conditions in the formulation. These are necessary in order to specify the initial state completely, so that a proper time response can be obtained. Thus the semi-infinite lossy line becomes the reference case for understanding the initialization of more general fractional order differential equations.

Assuming a lossy RC-line, the boundary value problem can be written in terms of the current or voltage variable [Karplus (1958)]. Since a semi-infinite line is considered, the measurable inputs or outputs are assumed to occur at the left end $(x=0)$, while the right end $(x=\infty)$ is kept at a finite value. The desired result is to mathematically define the behavior of the line at $x=0$, that is, to determine both the input impedance and the initialization response. In terms of the voltage variables, the problem can be written as

$$
\frac{\partial v(x, t)}{\partial t}=\alpha \frac{\partial^{2} v(x, t)}{\partial x^{2}}, v(0, t)=v_{I}(t), v(\infty, t)=0, v(x, 0) \text { given, }
$$

with

$$
i(x, t)=-\frac{1}{R} \frac{\partial v(x, t)}{\partial x}
$$

where $v$ is the voltage, $i$ is the current, $v_{I}(t)$ is a time-dependent input variable, and $\alpha$ is a constant which is one over the product of resistance per unit length, $R$, and capacitance per unit length, $C$. A classical solution using iterated Laplace transforms is used to solve this problem.

Taking the Laplace transform with respect to time and using s as the temporal Laplace variable gives 


$$
\mathrm{sV}(\mathrm{x}, \mathrm{s})-\mathrm{v}(\mathrm{x}, 0)=\alpha \frac{\mathrm{d}^{2} \mathrm{~V}(\mathrm{x}, \mathrm{s})}{\mathrm{dx}^{2}},
$$

with

$$
\mathrm{V}(0, \mathrm{~s})=\mathrm{V}_{\mathrm{I}}(\mathrm{s}), \quad \mathrm{V}(\infty, \mathrm{s})=0, \quad \mathrm{I}(\mathrm{x}, \mathrm{s})=\frac{-1}{\mathrm{R}} \frac{\mathrm{dV}(\mathrm{x}, \mathrm{s})}{\mathrm{dx}}
$$

Then taking the Laplace transform with respect to spatial position $\mathrm{x}$, and using $\mathrm{p}$ as the spatial Laplace variable gives

$$
\frac{\mathrm{s}}{\alpha} \mathrm{V}(\mathrm{p}, \mathrm{s})-\frac{1}{\alpha} \mathrm{V}(\mathrm{p}, 0)=\mathrm{p}^{2} \mathrm{~V}(\mathrm{p}, \mathrm{s})-\mathrm{pV}(0, \mathrm{~s})-\left[\frac{\mathrm{dV}}{\mathrm{dx}}\right]_{-(0, \mathrm{~s})} .
$$

Replacing $d V / d x$ with $V^{*}$, this equation can be manipulated to give $V(p, s)$ as

$$
\left[\mathrm{p}^{2}-\frac{\mathrm{s}}{\alpha}\right] \mathrm{V}(\mathrm{p}, \mathrm{s})=-\frac{1}{\alpha} \mathrm{V}(\mathrm{p}, 0)+\mathrm{pV}(0, \mathrm{~s})+\mathrm{V}^{*}(0, \mathrm{~s})
$$

or

$$
\mathrm{V}(\mathrm{p}, \mathrm{s})=\left[\frac{1}{\mathrm{p}^{2}-\frac{\mathrm{s}}{\alpha}}\right]\left[\frac{-1}{\alpha} \mathrm{V}(\mathrm{p}, 0)+\mathrm{pV}(0, \mathrm{~s})+\mathrm{V} *(0, \mathrm{~s})\right]
$$

Each term in this equation will be considered separately in order to inverse transform this with respect to the $p$ variable. The first term, the transform of the initial spatial distribution, $V(p, 0)$, is problem-dependent. A slight rearranging of the expression gives;

$$
\begin{aligned}
\mathrm{V}(\mathrm{p}, \mathrm{s})=\left[\frac{1}{2 \sqrt{\frac{\mathrm{s}}{\alpha}}\left(\mathrm{p}-\sqrt{\frac{\mathrm{s}}{\alpha}}\right)}-\frac{1}{2 \sqrt{\frac{\mathrm{s}}{\alpha}}\left(\mathrm{p}+\sqrt{\frac{\mathrm{s}}{\alpha}}\right)}\right]\left[\frac{-1}{\alpha} \mathrm{V}(\mathrm{p}, 0)\right] \\
+\left[\frac{1}{\mathrm{p}^{2}-\frac{\mathrm{s}}{\alpha}}\right]\left[\mathrm{pV}(0, \mathrm{~s})+\mathrm{V}^{*}(0, \mathrm{~s})\right] .
\end{aligned}
$$

Here the first term represents the effect of any initial spatial voltage distribution, while the second term represents the voltage and current present at the $x=0$ end of the line. The first term is now inverse-Laplace-transformed with respect to the $\mathrm{p}$ variable using convolution, and the other term is inverse-transformed using standard transform pairs; 


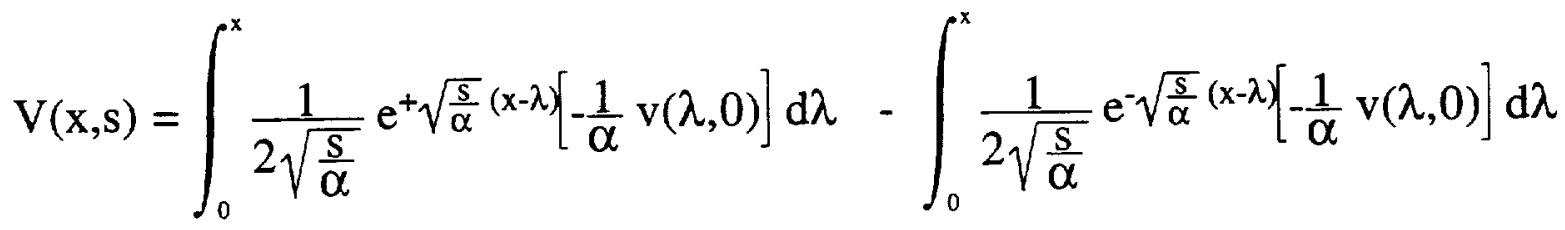

$$
\begin{aligned}
& +V(0, s) \cosh \left(\sqrt{\frac{s}{\alpha}} x\right)+\frac{V^{*}(0, s)}{\sqrt{\frac{s}{\alpha}}} \sinh \left(\sqrt{\frac{s}{\alpha}} x\right)
\end{aligned}
$$

Equivalently,

$$
\begin{aligned}
& \mathrm{V}(\mathrm{x}, \mathrm{s})=\int_{0}^{\mathrm{x}} \frac{1}{2 \sqrt{\frac{\mathrm{s}}{\alpha}}} \mathrm{e}^{+\sqrt{\frac{\mathrm{s}}{\alpha}}} \mathrm{x} \mathrm{e}^{-\sqrt{\frac{\mathrm{s}}{\alpha}}} \lambda\left[-\frac{1}{\alpha} \mathrm{v}(\lambda, 0)\right] \mathrm{d} \lambda \\
& -\int_{0}^{x} \frac{1}{2 \sqrt{\frac{s}{\alpha}}} e^{-\sqrt{\frac{s}{\alpha}}} x e^{+\sqrt{\frac{s}{\alpha}}} \lambda\left[-\frac{1}{\alpha} v(\lambda, 0)\right] d \lambda \\
& +\frac{V(0, s)}{2}\left[e^{+\sqrt{\frac{s}{\alpha}}} x+e^{-\sqrt{\frac{s}{\alpha}}} x\right]+\frac{V^{*}(0, s)}{2 \sqrt{\frac{s}{\alpha}}}\left[e^{+\sqrt{\frac{s}{\alpha}}} x-e^{-\sqrt{\frac{s}{\alpha}}} x\right]
\end{aligned}
$$

Collecting like exponentials then gives

$$
\begin{aligned}
\mathrm{V}(\mathrm{x}, \mathrm{s})= & \frac{\mathrm{e}^{+\sqrt{\frac{\mathrm{s}}{\alpha}}} \mathrm{x}}{2}\left[\mathrm{~V}(0, \mathrm{~s})+\frac{\mathrm{V}^{*}(0, \mathrm{~s})}{\sqrt{\frac{\mathrm{s}}{\alpha}}}-\frac{1}{\alpha \sqrt{\frac{\mathrm{s}}{\alpha}}} \int_{0}^{\mathrm{x}} \mathrm{e}^{-\sqrt{\frac{\mathrm{s}}{\alpha}} \lambda} \mathrm{v}(\lambda, 0) \mathrm{d} \lambda\right] \\
& +\frac{\mathrm{e}^{-\sqrt{\frac{\mathrm{s}}{\alpha}}} \mathrm{x}}{2}\left[\mathrm{~V}(0, \mathrm{~s})-\frac{\mathrm{V}^{*}(0, \mathrm{~s})}{\sqrt{\frac{\mathrm{s}}{\alpha}}}+\frac{1}{\alpha \sqrt{\frac{\mathrm{s}}{\alpha}}} \int_{0}^{\mathrm{x}} \mathrm{e}^{+\sqrt{\frac{\mathrm{s}}{\alpha}} \lambda} \mathrm{v}(\lambda, 0) \mathrm{d} \lambda\right] .
\end{aligned}
$$

It should be recognized that the coefficients multiplying the two exponential functions are unknowns. Although the integral and either $\mathrm{V}(0, \mathrm{~s})$ or $\mathrm{V}^{*}(0, \mathrm{~s})$ are given in the problem statement, the other condition ( $\mathrm{V}^{*}$ or $\mathrm{V}$, respectively) at $\mathrm{x}=0$ is determined as a response to these two given terms. Imposing the boundary condition at $\mathrm{x}=\infty$, allows the determination of a relationship between these three terms at $\mathrm{x}=0$, and thus allows the impedance and initial condition response of the system.

It is shown in the Appendix that in the limit as $x \rightarrow \infty$, the second term in Equation (14) goes to zero through application of L'Hospital's rule and Leibniz's rule, and by the fact that $V(\infty, s)=0$ from the problem statement. Then 


$$
V(\infty, s)=\frac{e^{+\sqrt{\frac{s}{\alpha}} \infty}}{2}\left[V(0, s)+\frac{V^{*}(0, s)}{\sqrt{\frac{s}{\alpha}}}-\operatorname{Lim}_{x \rightarrow \infty} \frac{1}{\alpha \sqrt{\frac{s}{\alpha}}} \int_{0}^{x} e^{-\sqrt{\frac{s}{\alpha}} \lambda} v(\lambda, 0) d \lambda\right]=0 .
$$

Dropping the limit notation, by necessity we have

$$
\mathrm{V}(0, \mathrm{~s})+\frac{\mathrm{V}^{*}(0, \mathrm{~s})}{\sqrt{\frac{\mathrm{s}}{\alpha}}}-\frac{1}{\alpha \sqrt{\frac{\mathrm{s}}{\alpha}}} \int_{0}^{\infty} \mathrm{e}^{-\sqrt{\frac{\mathrm{s}}{\alpha}}} \lambda \mathrm{v}(\lambda, 0) \mathrm{d} \lambda=0
$$

Remembering that the current anywhere in the line is related to the voltage, then at $x=0$

$$
\mathrm{I}(0, \mathrm{~s})=-\frac{1}{\mathrm{R}} \frac{\mathrm{dV}(0, \mathrm{~s})}{\mathrm{dx}}=-\frac{\mathrm{V}^{*}(0, \mathrm{~s})}{\mathrm{R}},
$$

and solving for voltage in terms of source current in Equation (16) gives

$$
\mathrm{V}(0, \mathrm{~s})=\frac{\mathrm{R} \mathrm{I}(0, \mathrm{~s})}{\sqrt{\frac{\mathrm{s}}{\alpha}}}+\frac{1}{\alpha \sqrt{\frac{\mathrm{s}}{\alpha}}} \int_{0}^{\infty} \mathrm{e}^{-\sqrt{\frac{\mathrm{s}}{\alpha}} \lambda} \mathrm{v}(\lambda, 0) \mathrm{d} \lambda .
$$

In evaluating the integral on the right, it is now recognized that this term is equivalent to a Laplace transform integral with the Laplace operator $\sqrt{\frac{S}{\alpha}}$. Thus, Laplace transform tables can simplify the evaluation of this term as follows:

$$
\frac{1}{\alpha \sqrt{\frac{s}{\alpha}}} \int_{0}^{\infty} \mathrm{e}^{-\sqrt{\frac{s}{\alpha}} \lambda} \mathrm{v}(\lambda, 0) \mathrm{d} \lambda=\frac{1}{\alpha \sqrt{\frac{\mathrm{s}}{\alpha}}}[\mathrm{V}(\mathrm{p}, 0)]_{\mathrm{F}=\sqrt{\frac{\mathrm{s}}{\alpha}}}
$$

The notation on the right side of this equation is used to indicate the evaluation procedure. First the initial spatial distribution $v(x, 0)$ is Laplace transformed with respect to the spatial Laplace variable $p$ to give $V(p, 0)$. The integral on the left of Equation (18) is then easily evaluated by replacing the spatial Laplace variable $p$ with $\sqrt{\mathrm{s} / \alpha}$. Using this, Equation (17) becomes

$$
\mathrm{V}(0, \mathrm{~s})=\frac{\mathrm{R} \mathrm{I}(0, \mathrm{~s})}{\sqrt{\frac{\mathrm{s}}{\alpha}}}+\frac{1}{\alpha \sqrt{\frac{\mathrm{s}}{\alpha}}}[\mathrm{V}(\mathrm{p}, 0)]_{\mathrm{p}=\sqrt{\frac{\mathrm{s}}{\alpha}}}
$$

Notice that this contains the driving point impedance functic $\mathrm{Z}(\mathrm{s})$, which is obtained by setting the initial condition terms to zero,

$$
Z(s)=\frac{V(0, s)}{I(0, s)}=\frac{R}{\sqrt{\frac{s}{\alpha}}}
$$


or, as $\alpha=1 / \mathrm{RC}$,

$$
\mathrm{Z}(\mathrm{s})=\frac{\mathrm{V}(0, \mathrm{~s})}{\mathrm{I}(0, \mathrm{~s})}=\sqrt{\frac{\mathrm{R}}{\mathrm{C}}} \frac{1}{\sqrt{\mathrm{s}}}
$$

Notice from Equation (19) that the voltage $V(0, s)$ is seen to be composed of two parts, the forced response due to $\mathrm{I}(0, \mathrm{~s})$ and the initial condition response due to the initial voltage distribution in the lossy line.

Applying the other boundary condition of Equation (7) at $x=0$, allows the solution of the voltage transfer function $\mathrm{V}(\mathrm{x}, \mathrm{s}) / \mathrm{V}(0, \mathrm{~s})$ and has no effect on the terminal impedance. This is now done for completeness. At $x=0$, the boundary condition is

$$
\mathrm{V}(0, \mathrm{~s})=\mathrm{V}_{\mathrm{I}}(\mathrm{s}) \text {. }
$$

Now, inserting Equations (21), (19), and (16') into Equation (14), gives

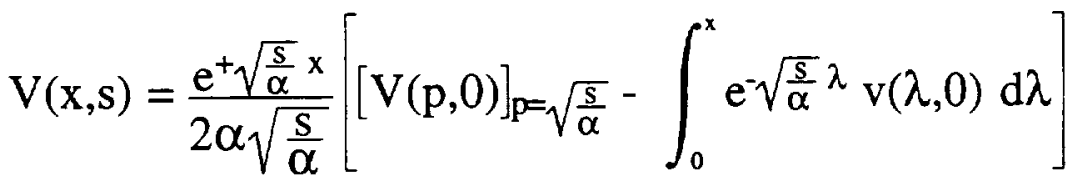

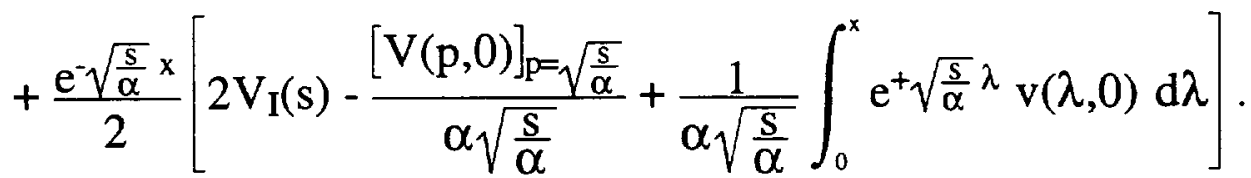

This equation is the general expression for the voltage anywhere inside the line as a function of the applied voltage at the terminal $V_{I}(s)$ and the initial conditions on the line. Furthermore, the current at any point in the line can be determined directly from Equation (22) as

$$
I(x, s)=-\frac{1}{R} \frac{d V(x, s)}{d x}
$$

We will now discuss the voltage-current behavior of the lossy line. Referring back to the definition of the Laplace transform from Equation (4), and applying it to Equation (19), it can be seen that the input-output properties (with initial conditions equal to zero) of the lossy line satisfy

$$
\mathrm{i}(\mathrm{t})=\frac{1}{\mathrm{R} \sqrt{\alpha}} \frac{\mathrm{d}^{1 / 2} \mathrm{v}(\mathrm{t})}{\mathrm{dt}^{1 / 2}} \text { or } \mathrm{v}(\mathrm{t})=\mathrm{R} \sqrt{\alpha} \frac{\mathrm{d}^{-1 / 2} \mathrm{i}(\mathrm{t})}{\mathrm{dt}^{-1 / 2}}
$$

More completely, from Equation (17) or (19),

$$
\mathrm{v}(\mathrm{t})=\mathrm{R} \sqrt{\alpha} \frac{\mathrm{d}^{-1 / 2} \mathrm{i}(\mathrm{t})}{\mathrm{dt}^{-1 / 2}}+\phi_{1}(\mathrm{t})
$$


where $\phi_{1}(t)$ is obtained as the inverse Laplace transform of either term in Equation (18). Likewise,

$$
i(t)=\frac{1}{R \sqrt{\alpha}} \frac{d^{1 / 2} v(t)}{{d t^{1 / 2}}^{1 / 2}}+\phi_{2}(t)
$$

where $\phi_{2}(t)$ is effectively the negative integral of $\phi_{1}(t)$ weighted by $1 / R \sqrt{\alpha}$. From these expressions it should be clear that the fractional differintegral of any function must include a timedependent initial condition term.

\section{The Semi-Infinite Lossless Line}

This section considers the semi-infinite lossless line whose impedance is a constant or an operator of order zero. A classical iterated Laplace transform is again used for the derivation of the operational impedance for the semi-infinite lossless line. The inclusion of the spatial initial conditions in the formulation is of particular importance. These are necessary in order to specify the initial state completely, so that a proper time response can be obtained. The results will aid in the understanding of the initial condition terms for integer order systems in a fractional ordinary differential equation setting.

Assuming a lossless LC-line, the boundary value problem can be written in terms of the current or voltage variable [Karplus (1958)]. Since a semi-infinite line is considered, the measurable inputs or outputs are assumed to occur at the left end $(x=0)$, while the right end $(x=\infty)$ must be kept at a finite value. The desired result is an impedance at the left end of the line as well as the response due to any initial conditions on the line. In terms of the voltage variables, the problem can be written as

$$
\begin{aligned}
\frac{\partial^{2} v(x, t)}{\partial t^{2}}= & \frac{1}{L C} \frac{\partial^{2} v(x, t)}{\partial x^{2}}, \\
& v(0, t)=v_{I}(t), v(\infty, t)=0, v(x, 0) \text { and } v^{\prime}(x, 0) \text { given, }
\end{aligned}
$$

with

$$
\frac{\partial \mathrm{i}(\mathrm{x}, \mathrm{t})}{\partial \mathrm{x}}=-\mathrm{C} \frac{\partial \mathrm{v}(\mathrm{x}, \mathrm{t})}{\partial \mathrm{t}} \text { and } \frac{\partial \mathrm{v}(\mathrm{x}, \mathrm{t})}{\partial \mathrm{x}}=-\mathrm{L} \frac{\partial \mathrm{i}(\mathrm{x}, \mathrm{l})}{\partial \mathrm{t}}
$$

where $v$ is the voltage, $i$ is the current, $v_{I}(t)$ is a time-dependent input variable, $L$ is inductance per unit length, and $C$ is capacitance per unit length. A classizal solution to this problem is obtained using iterated Laplace transforms. 
Taking the Laplace transform with respect to time and using $s$ as the temporal Laplace variable gives

$$
s^{2} V(x, s)-s v(x, 0)-\frac{d v(x, 0)}{d t}=\frac{1}{L C} \frac{d^{2} V(x, s)}{d^{2}}
$$

with boundary conditions

$$
\begin{aligned}
& \mathrm{V}(0, \mathrm{~s})=\mathrm{V}_{\mathrm{I}}(\mathrm{s}), \quad \mathrm{V}(\infty, \mathrm{s})=0, \\
& \left.\frac{\mathrm{dV}(\mathrm{x}, \mathrm{s})}{\mathrm{dx}}=-\mathrm{L}\left(L \mid \frac{\mathrm{dI}(\mathrm{x}, \mathrm{t})}{\mathrm{dt}}\right\}\right)=-\mathrm{L}[\mathrm{sI}(\mathrm{x}, \mathrm{s})-\mathrm{I}(\mathrm{x}, 0)] .
\end{aligned}
$$

Then taking the Laplace transform with respect to spatial position, $\mathrm{x}$, and using $\mathrm{p}$ as the spatial Laplace variable gives

$$
\operatorname{LCs}^{2} \mathrm{~V}(\mathrm{p}, \mathrm{s})-\operatorname{LCsV}(\mathrm{p}, 0)-\mathrm{LCV}^{\prime}(\mathrm{p}, 0)=\mathrm{p}^{2} \mathrm{~V}(\mathrm{p}, \mathrm{s})-\mathrm{pV}(0, \mathrm{~s})-\left[\frac{\mathrm{dV}}{\mathrm{dx}}\right]_{(0, \mathrm{~s})},
$$

where $d v(x, 0) / d t$ is replaced with $V^{\prime}$. Further replacing $d V(0, s) / d x$ with $V^{*}$, this equation can be manipulated to give $\mathrm{V}(\mathrm{p}, \mathrm{s})$ as

$$
\left[p^{2}-L_{C}^{2}\right] V(p, s)=-\operatorname{LCsV}(p, 0)-L C V^{\prime}(p, 0)+p V(0, s)+V^{*}(0, s)
$$

or

$$
\mathrm{V}(\mathrm{p}, \mathrm{s})=\left[\frac{1}{\mathrm{p}^{2}-\mathrm{LCs} \mathrm{s}^{2}}\right]\left[-\mathrm{LCs} \mathrm{V}(\mathrm{p}, 0)-\mathrm{LC} \mathrm{V}^{\prime}(\mathrm{p}, 0)+\mathrm{pV}(0, \mathrm{~s})+\mathrm{V}^{*}(0, \mathrm{~s})\right] .
$$

Each term in this equation will be considered separately in order to inverse-Laplace- transform this with respect to the $p$ variable. The first term, the initial spatial distribution $V(p, 0)$, is problemdependent. A slight rearranging of the expression gives

$$
\begin{gathered}
\mathrm{V}(\mathrm{p}, \mathrm{s})=\left[\frac{1}{2 \mathrm{~s} \sqrt{\mathrm{LC}}(\mathrm{p}-\mathrm{s} \sqrt{\mathrm{LC}})}-\frac{1}{2 \mathrm{~s} \sqrt{\mathrm{LC}}(\mathrm{p}+\mathrm{s} \sqrt{\mathrm{LC}})}\right]\left[-\mathrm{LCs} \mathrm{V}(\mathrm{p}, 0)-\mathrm{LC} \mathrm{V}^{\prime}(\mathrm{p}, 0)\right] \\
+\left[\frac{1}{\mathrm{p}^{2}-\mathrm{LCs}^{2}}\right]\left[\mathrm{pV}(0, \mathrm{~s})+\mathrm{V}^{*}(0, \mathrm{~s})\right]
\end{gathered}
$$

Here the first term represents the effect of any initial spatial distribution, while the second term represents the voltage and current at the $x=0$ end of the line. The first term is now inverseLaplace-transformed with respect to the $\mathrm{p}$ variable using convolution, and the other terms are inverse-transformed using standard transform pairs; 


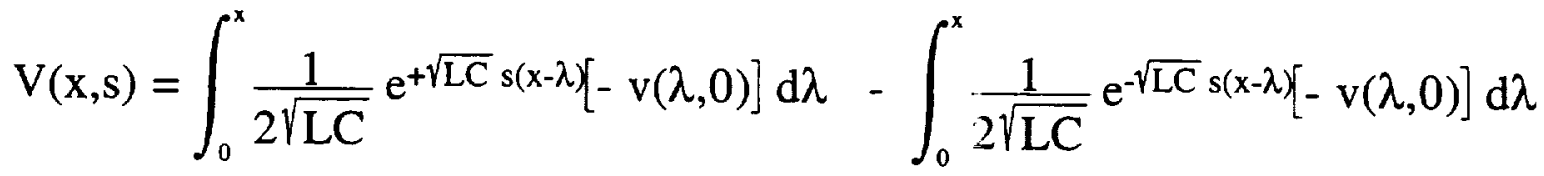

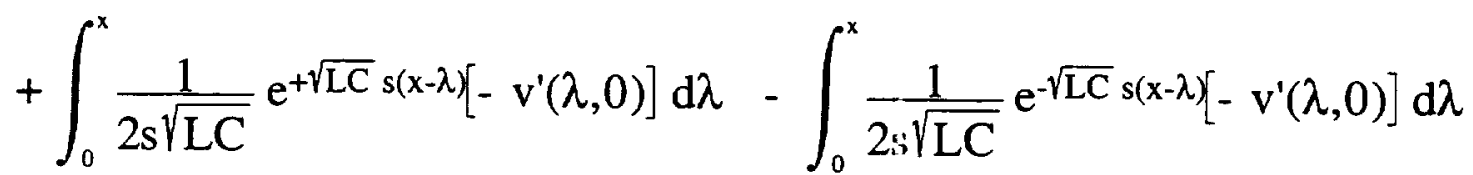

$$
\begin{aligned}
& +\mathrm{V}(0, \mathrm{~s}) \cosh (\sqrt{\mathrm{LC}} \mathrm{sx})+\frac{\mathrm{V}^{*}(0, \mathrm{~s})}{\sqrt{\mathrm{LC}} \mathrm{s}} \sinh (\sqrt{\mathrm{LC}} \mathrm{sx})
\end{aligned}
$$

Collecting like exponentials then gives

$$
\begin{aligned}
V(x, s)= & \frac{e^{+\sqrt{L C} s x}}{2}\left[V(0, s)+\frac{V^{*}(0, s)}{\sqrt{L C} s}\right. \\
& \left.-\frac{1}{\sqrt{L C}} \int_{0}^{x} e^{-\sqrt{L C}} s \lambda v(\lambda, 0) d \lambda-\frac{1}{s \sqrt{L C}} \int_{0}^{x} e^{-\sqrt{L C} s \lambda} v^{\prime}(\lambda, 0) d \lambda\right] \\
+ & \frac{e^{-\sqrt{L C}} s x}{2}\left[V(0, s)-\frac{V^{*}(0, s)}{\sqrt{L C} s}\right. \\
& \left.+\frac{1}{\sqrt{L C}} \int_{0}^{x} e^{+\sqrt{L C}} s \lambda v(\lambda, 0) d \lambda+\frac{1}{s \sqrt{L C}} \int_{0}^{x} e^{+\sqrt{L C}} s \lambda v^{\prime}(\lambda, 0) d \lambda\right] .
\end{aligned}
$$

It should be recognized that the coefficients multiplying the two exponential functions are unknowns. Although the integrals and either $\mathrm{V}(0, \mathrm{~s})$ or $\mathrm{V}^{*}(\mathrm{O}, \mathrm{s})$ are given in the problem statement, the other condition ( $\mathrm{V}^{*}$ or $\mathrm{V}$, respectively) at $\mathrm{x}=0$ is detennined as a response to the given terms. Imposing the boundary condition at $\mathrm{x}=\infty$, allows the deternination of a relationship between these three terms at $x=0$, and thus the impedance and initial condition response of the system.

As was shown for Equation (14), in the limit as $x \rightarrow \infty$, the second term in Equation (33) goes to zero through application of L'Hospital's rule and Leibniz's rule, and by the fact that $V(\infty, s)=0$ from the problem statement. Then

$$
\begin{aligned}
\mathrm{V}(\infty, \mathrm{s}) \equiv 0 & =\frac{\mathrm{e}^{+\sqrt{\mathrm{LC}} \mathrm{s} \infty}}{2}\left[\mathrm{~V}(0, \mathrm{~s})+\frac{\mathrm{V}^{*}(0, \mathrm{~s})}{\sqrt{\mathrm{LC}} \mathrm{s}}\right. \\
& \left.-\frac{1}{\sqrt{\mathrm{LC}}} \int_{0}^{\infty} \mathrm{e}^{-\sqrt{\mathrm{LC}} \mathrm{s} \lambda} \mathrm{v}(\lambda, 0) \mathrm{d} \lambda-\frac{1}{\mathrm{~s} \sqrt{\mathrm{LC}}} \int_{0}^{\infty} \mathrm{e}^{-\sqrt{\mathrm{LC}} \mathrm{s} \lambda} \mathrm{v}^{\prime}(\lambda, 0) \mathrm{d} \lambda\right],
\end{aligned}
$$


and by necessity,

$$
\begin{aligned}
V(0, s)+\frac{V^{*}(0, s)}{\sqrt{L C} s}-\frac{1}{\sqrt{L C}} \int_{0}^{\infty} & e^{-\sqrt{L C} s \lambda} v(\lambda, 0) d \lambda \\
& \quad-\frac{1}{s \sqrt{L C}} \int_{0}^{\infty} e^{-\sqrt{L C}} s \lambda v^{\prime}(\lambda, 0) d \lambda=0 .
\end{aligned}
$$

Solving for the voltage in terms of the voltage gradient in Equation (35) gives

$$
\begin{aligned}
V(0, s)=-\frac{V^{*}(0, s)}{\sqrt{L C} s}+\frac{1}{\sqrt{L C}} & \int_{0}^{\infty} e^{-\sqrt{L C}} s \lambda v(\lambda, 0) d \lambda \\
& +\frac{1}{s \sqrt{L C}} \int_{0}^{\infty} e^{-\sqrt{L C}} s^{2} v^{\prime}(\lambda, 0) d \lambda .
\end{aligned}
$$

In evaluating the integrals, it is now recognized that these terms are equivalent to a Laplace transform integral with the Laplace operator $\sqrt{\mathrm{LC}} \mathrm{s}$. Thus Laplace transform tables can simplify the evaluation of the third term as follows:

$$
\frac{1}{\sqrt{\mathrm{LC}}} \int_{0}^{\infty} \mathrm{e}^{-\sqrt{\mathrm{LC}} \mathrm{s} \lambda} \mathrm{v}(\lambda, 0) \mathrm{d} \lambda=\frac{1}{\sqrt{\mathrm{LC}}}[\mathrm{V}(\mathrm{p}, 0)]_{\mathrm{p}=\sqrt{\mathrm{LC}} \mathrm{s}}
$$

and similarly the fourth term. The notation on the right side of this equation is used to indicate the evaluation procedure. First the initial spatial distribution, $v(x, 0)$, is Laplace transformed with respect to the spatial Laplace variable $p$ to give $V(p, 0)$. The integral on the left of Equation (37) is then easily evaluated by replacing the spatial Laplace variable $\mathrm{p}$ with $\sqrt{\mathrm{LC}} \mathrm{s}$. Using this, Equation (36) becomes

$$
\mathrm{V}(0, \mathrm{~s})=\frac{-\mathrm{V}^{*}(0, \mathrm{~s})}{\sqrt{\mathrm{LC}} \mathrm{s}}+\frac{1}{\sqrt{\mathrm{LC}}}[\mathrm{V}(\mathrm{p}, 0)]_{\mathrm{p}=\sqrt{\mathrm{LC}} \mathrm{s}}+\frac{1}{\sqrt{\mathrm{LC}} \mathrm{s}}\left[\mathrm{V}^{\prime}(\mathrm{p}, 0)\right]_{\mathrm{p}=\sqrt{\mathrm{LC}} \mathrm{s}}
$$

This contains the driving point transfer function (not an impedance),

$$
\frac{V(0, s)}{V^{*}(0, s)}=-\frac{1}{\sqrt{L C} s}
$$


which is obtained by setting the initial condition terms to zero. Notice from Equation (38) that the voltage $V(0, s)$ is seen to be composed of two parts, the forced response due to $V^{*}(0, s)$ and the initial condition response due to the initial voltage distribution in the lossless line. Mathematically this is expressed in the time domain as

$$
v(0, t)=-\frac{1}{\sqrt{L C}} \int \frac{d v(0, t)}{d x} d t+\phi_{3}(t),
$$

where $\phi_{3}(t)$ is the time-dependent initial condition response due to the initial voltage and current distributions on the line, which can be obtained by inverse-Laplace-transforming the two integrals in Equation (36). Thus it is seen that a simple integral operator can also have time varying initial condition terms.

More importantly, notice that Equation (38) contains $V^{*}(0, s)$. From the boundary conditions given in Equation (27), this term can be replaced with

$$
\left[\frac{\mathrm{dV}(\mathrm{x}, \mathrm{s})}{\mathrm{dx}}\right]_{\mathrm{x}=0}=-\mathrm{L}[\mathrm{sI}(0, \mathrm{~s})-\mathrm{I}(0,0)] .
$$

Equation (38) then becomes

$$
\mathrm{V}(0, \mathrm{~s})=\frac{\mathrm{L}[\mathrm{sI}(0, \mathrm{~s})-\mathrm{I}(0,0)]}{\sqrt{\mathrm{LC}} \mathrm{s}}+\frac{1}{\sqrt{\mathrm{LC}}}[\mathrm{V}(\mathrm{p}, 0)]_{\mathrm{p}=1 \overline{\mathrm{LC}} \mathrm{s}}+\frac{1}{\sqrt{\mathrm{LC}} \mathrm{s}}\left[\mathrm{V}^{\prime}(\mathrm{p}, 0)\right]_{\mathrm{p}=\sqrt{\mathrm{LC}} \mathrm{s}}
$$

or

$$
\begin{aligned}
\mathrm{V}(0, \mathrm{~s})=\sqrt{\frac{\mathrm{L}}{\mathrm{C}}} & \mathrm{I}(0, \mathrm{~s})-\sqrt{\frac{\mathrm{L}}{\mathrm{C}}} \frac{[\mathrm{I}(0,0)]}{\mathrm{s}} \\
& +\frac{1}{\sqrt{\mathrm{LC}}}[\mathrm{V}(\mathrm{p}, 0)]_{\mathrm{p}=\sqrt{\mathrm{LC}} \mathrm{s}}+\frac{1}{\sqrt{\mathrm{LC}} \mathrm{s}}\left[\mathrm{V}^{\prime}(\mathrm{p}, 0)\right]_{\mathrm{p}=\sqrt{\mathrm{LC}} \mathrm{s}}
\end{aligned}
$$

Notice from Equation (42) that the voltage $V(0, s)$ is seen to be composed of two parts, the forced response due to $I(0, s)$ and the initial condition response due to the initial voltage distribution in the lossless line. Considering only the first term, it can be seen that the impedance looking into this line is then

$$
\mathrm{Z}(\mathrm{s})=\frac{\mathrm{V}(0, \mathrm{~s})}{\mathrm{I}(0, \mathrm{~s})}=\sqrt{\frac{\mathrm{L}}{\mathrm{C}}}
$$

which is simply a constant. Mathematically, Equation (42):s expressed in the time domain as 


$$
\mathrm{v}(0, \mathrm{t})=\sqrt{\frac{\mathrm{L}}{\mathrm{C}} \mathrm{i}(0, \mathrm{t})+\phi_{4}(\mathrm{t})}
$$

where this $\phi_{4}(t)$ is the time-dependent initial condition response due to the initial voltage and current distributions on the line, which can be obtained by inverse-Laplace- transforming the three initial condition terms in Equation (42). Thus it is seen that even a simple constant gain operator can also have time-varying initial condition terms.

\section{The Semi-Infinite Vibrating Bar}

This section considers longitudinal vibrations in the semi-infinite elastic bar which has a transfer function that is a pure integral of order unity. A classical iterated Laplace transform is again used for the derivation of the operational impedance for the semi-infinite elastic bar. The inclusion of the spatial initial conditions in the formulation is of particular importance. These are necessary in order to specify the initial state completely, so that a proper time response can be obtained. The results will aid in the understanding of the initial condition terms for integer order systems in a fractional ordinary differential equation setting.

For the elastic bar, the boundary value problem can be written in terms of the longitudinal displacement and the longitudinal force [Churchill (1958)]. Since a semi-infinite bar is considered, the measurable inputs or outputs are assumed to occur at the left end $(x=0)$, while the right end $(x=\infty)$ must be kept stationary. The desired result is an impedance at the left end of the bar as well as the response due to any initial conditions on the bar. In terms of the displacement $y(x, t)$, the problem can be written as

$$
\frac{\partial^{2} y(x, t)}{\partial t^{2}}=\frac{E}{\rho} \frac{\partial^{2} y(x, t)}{\partial x^{2}}, y(\infty, t)=0, y(x, 0) \text { and } y^{\prime}(x, 0) \text { given, }
$$

with

$$
-E \frac{\partial y(0, t)}{\partial x}=f(t)
$$

where $E$ is Young's modulus of elasticity, $\rho$ is the mass per unit volume, $f(t)$ is the force at the left end of the bar, and $\partial y / \partial x$ is the strain which is proportional to the force. The force can be the applied input with the displacement as the output, or the displacement can be the input with the force as the output, as desired by the user. In what follows we will let $a^{2}=E / \rho$.

A classical solution using iterated Laplace transforms will be used to solve this problem. The results from this solution will provide further guidance into fractional ordinary differential equations.

Taking the Laplace transform with respect to time and using $s$ as the temporal Laplace variable gives 


$$
s^{2} Y(x, s)-s y(x, 0)-\frac{d y(x, 0)}{d t}=a^{2} \frac{d^{2} Y(x, s)}{d x^{2}},
$$

with

$$
\mathrm{Y}(\infty, \mathrm{s})=0, \quad \frac{\mathrm{dY}(0, \mathrm{~s})}{\mathrm{dx}}=-\frac{1}{\mathrm{E}} \mathrm{F}(\mathrm{s})=\mathrm{Y}^{*}(0, \mathrm{~s}) .
$$

Then taking the Laplace transform with respect to spatial position $\mathrm{x}$ and using $\mathrm{p}$ as the spatial Laplace variable gives

$$
\frac{1}{a^{2}} s^{2} Y(p, s)-\frac{1}{a^{2}} s Y(p, 0)-\frac{1}{a^{2}} Y^{\prime}(p, 0)=p^{2} Y(p, s)-p Y(0, s)-\left[\frac{d Y}{d x}\right]_{(0, s)} .
$$

Replacing $d Y(x, s) / d x$ with $Y^{*}$ and $d y(x, 0) / d t$ by $y^{\prime}$, this equation can be manipulated to give $\mathrm{Y}(\mathrm{p}, \mathrm{s})$ as

$$
\left[p^{2}-\frac{1}{a^{2}} s^{2}\right] Y(p, s)=-\frac{1}{a^{2}} s Y(p, 0)-\frac{1}{a^{2}} Y^{\prime}(p, 0)+p Y(0, s)+Y^{*}(0, s)
$$

or

$$
\mathrm{Y}(\mathrm{p}, \mathrm{s})=\left[\frac{1}{\mathrm{p}^{2}-\frac{1}{\mathrm{a}^{2}} \mathrm{~s}^{2}}\right]\left[-\frac{1}{\mathrm{a}^{2}} \mathrm{~s} \mathrm{Y}(\mathrm{p}, 0)-\frac{1}{\mathrm{a}^{2}} \mathrm{Y}^{\prime}(\mathrm{p}, 0)+\mathrm{pY}(0, \mathrm{~s})+\mathrm{Y}^{*}(0, \mathrm{~s})\right] .
$$

Each term in this equation will be considered separately in order to inverse-Laplace- transform this with respect to the $\mathrm{p}$ variable. The first term, the initial spatial distribution $\mathrm{Y}(\mathrm{p}, 0)$, is problemdependent. A slight rearranging of the expression gives

$$
\begin{gathered}
Y(p, s)=\left[\frac{1}{\frac{2 s}{a}\left(p-\frac{s}{a}\right)}-\frac{1}{\frac{2 s}{a}\left(p+\frac{s}{a}\right)}\right]\left[-\frac{1}{a^{2}} s Y\left(p, 0,-\frac{1}{a^{2}} Y^{\prime}(p, 0)\right]\right. \\
+\left[\frac{1}{p^{2}-\frac{s^{2}}{a^{2}}}\right]\left[p Y(0, s)+Y^{*}(0, s)\right] .
\end{gathered}
$$

Here the first term represents the effect of any initial spatial distribution, while the second term represents the relationship of the displacement and force at : he $\mathrm{x}=0$ end of the bar. The first term is now inverse-Laplace-transformed with respect to the $p$ va-iable using convolution, and the other term is inverse-transformed using standard transform pairs; 


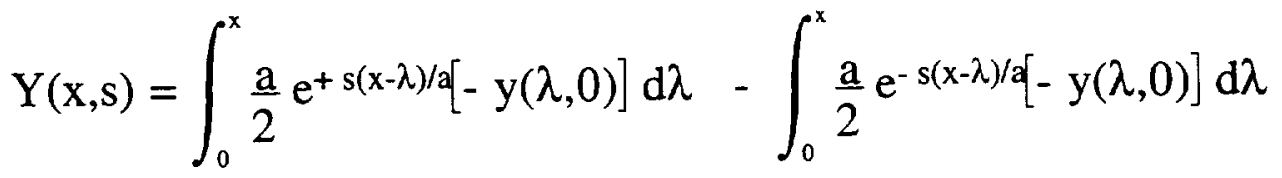

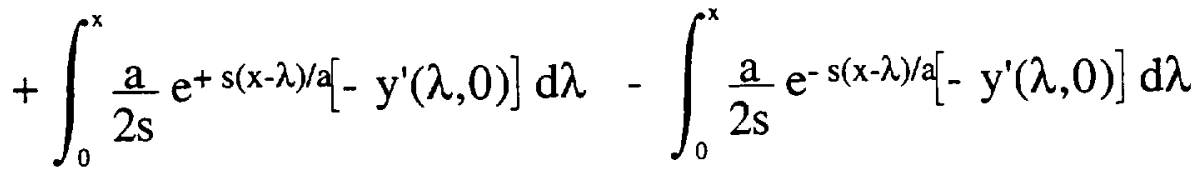

$$
\begin{aligned}
& +Y(0, s) \cosh \left(\frac{s x}{a}\right)+\frac{a Y^{*}(0, s)}{s} \sinh \left(\frac{s x}{a}\right) .
\end{aligned}
$$

Collecting like exponentials then gives

$$
\begin{aligned}
& Y(x, s)= \frac{e^{+s x / a}}{2}\left[Y(0, s)+\frac{a Y^{*}(0, s)}{s}\right. \\
&\left.-a \int_{0}^{x} e^{-s \lambda / a} y(\lambda, 0) d \lambda-\frac{a}{s} \int_{0}^{x} e^{-s \lambda / a} y^{\prime}(\lambda, 0) d \lambda\right] \\
&+\frac{e^{-s x / a}}{2}\left[Y(0, s)-\frac{a Y^{*}(0, s)}{s}\right. \\
&\left.\quad+a \int_{0}^{x} e^{+s \lambda / a} v(\lambda, 0) d \lambda+\frac{a}{s} \int_{0}^{x} e^{+s \lambda / a} y^{\prime}(\lambda, 0) d \lambda\right]
\end{aligned}
$$

It should be recognized that the coefficients multiplying the two exponential functions are unknowns. Although the integrals and either $\mathrm{Y}(0, \mathrm{~s})$ or $\mathrm{Y}^{*}(0, \mathrm{~s})$ are given in the problem statement, the other condition ( $\mathrm{Y}^{*}$ or $\mathrm{Y}$, respectively) at $\mathrm{x}=0$ is determined as a response to the given terms. Imposing the boundary condition at $\mathrm{x}=\infty$, allows the determination of a relationship between these three terms at $\mathrm{x}=0$, and thus allows the impedance and initial condition response of the system.

As was shown for Equation (14), in the limit as $x \rightarrow \infty$, the second term in Equation (53) goes to zero through application of L'Hospital's rule and Leibniz's rule, and by the fact that $Y(\infty, s)=0$ from the problem statement. Then

$$
\begin{aligned}
& \mathrm{Y}(\infty, \mathrm{s}) \equiv 0=\frac{\mathrm{e}^{+\mathrm{s} \infty / \mathrm{a}}}{2}\left[\mathrm{Y}(0, \mathrm{~s})+\frac{\mathrm{aY}(0, \mathrm{~s})}{\mathrm{s}}\right. \\
&\left.-\mathrm{a} \int_{0}^{\infty} \mathrm{e}^{-\mathrm{s} \lambda / \mathrm{a}} \mathrm{y}(\lambda, 0) \mathrm{d} \lambda-\frac{\mathrm{a}}{\mathrm{s}} \int_{0}^{\infty} \mathrm{e}^{-\mathrm{s} \lambda / \mathrm{a}} \mathrm{y}^{\prime}(\lambda, 0) \mathrm{d} \lambda\right],
\end{aligned}
$$


and by necessity,

$$
Y(0, s)+\frac{a Y^{*}(0, s)}{s}-a \int_{0}^{\infty} e^{-s \lambda / a} y(\lambda, 0) d \lambda-\frac{a}{s} \int_{0}^{\infty} e^{-s \lambda / a} y^{\prime}(\lambda, 0) d \lambda=0
$$

Solving for the displacement in terms of the strain (or force) in Equation (55) gives

$$
Y(0, s)=-\frac{a Y^{*}(0, s)}{s}+a \int_{0}^{\infty} e^{-s \lambda / a} y(\lambda, 0) d \lambda+\frac{a}{s} \int_{0}^{\infty} e^{-s \lambda / a} y^{\prime}(\lambda, 0) d \lambda
$$

In evaluating the integrals, it is now recognized that these terms are equivalent to a Laplace transform integral with the Laplace operator s/a. Thus, Laplace transform tables can simplify the evaluation of the third term as follows:

$$
\int_{0}^{\infty} e^{-s \lambda / a} y(\lambda, 0) d \lambda=a[Y(p, 0)]_{p=s / a}
$$

and similarly, the fourth term. The notation on the right side of this equation is used to indicate the evaluation procedure. First the initial spatial distribution, $y(x, 0)$, is Laplace transformed with respect to the spatial Laplace variable $p$ to give $Y(p, 0)$. The integral on the left of Equation (57) is then easily evaluated by replacing the spatial Laplace variable $\mathrm{p}$ with s/a. Using this, Equation (56) becomes

$$
Y(0, s)=\frac{-a Y^{*}(0, s)}{s}+a[Y(p, 0)]_{p=s / a}+\frac{a}{s}\left[Y^{\prime}(p, 0)\right]_{p=s / a}
$$

and this contains the transfer function, which is not an impedance,

$$
\frac{\mathrm{Y}(0, \mathrm{~s})}{\mathrm{Y}^{*}(0, \mathrm{~s})}=-\frac{\mathrm{a}}{\mathrm{s}}
$$

Notice from Equation (58) that the displacement $Y(0, s)$ is seen to be composed of two parts, the forced response due to the applied strain and the initial condition response due to the initial displacement distribution in the elastic bar. Mathematically this is expressed in the time domain as

$$
y(0, t)=-a \int \frac{d y(0, t)}{d x} d t+\phi_{5}(t)
$$

where $\phi_{5}(t)$ is the time dependent initial condition res\}onse due to the initial displacement 
distribution in the elastic bar, which can be obtained by inverse-Laplace-transforming the two integrals in Equation (56). Thus it is seen that a simple integral operator can also have timevarying initial condition terms.

Likewise, if the input at the left end was displacement, the force (strain) response can also be determined from Equation (58);

$$
\mathrm{Y}^{*}(0, \mathrm{~s})=-\frac{\mathrm{s}}{\mathrm{a}} \mathrm{Y}(0, \mathrm{~s})+[\mathrm{Y}(\mathrm{p}, 0)]_{\mathrm{p}=\mathrm{s} / \mathrm{a}}+\left[\mathrm{Y}^{\prime}(\mathrm{p}, 0)\right]_{\mathrm{p}=\mathrm{s} / \mathrm{a}}
$$

The strain response $\mathrm{Y}^{*}(0, \mathrm{~s})$ is then composed of two parts the forced response due to the applied displacement $\mathrm{Y}(0, \mathrm{~s})$ and the initial condition response due to any displacement distribution on the bar at time zero. In the time domain, this is

$$
y^{*}(0, t)=-\frac{1}{a} \frac{d y(0, t)}{d t}+\phi_{6}(t)
$$

Again $\phi_{6}(t)$ can be obtained from the given initial displacements.

\section{Summary}

This paper has demonstrated that when specific differintegral operators ( $q=1 / 2, q=1$, and $q=0$ ) are considered as semi-infinite systems, a time-dependent term resulting from the initial spatial conditions should be added to the to the forced response. This is an important result with respect to the solution of fractional ordinary differential equations that can have rather arbitrary initial conditions.

To apply this result to a specific fractional order differential equation, the initial conditions, if any, must be given along with some structural information on how they belong in the problem. For example, if the following system were given,

$$
\frac{\mathrm{d}^{3 / 2} \mathrm{f}(\mathrm{t})}{\mathrm{dt}^{3 / 2}}+\frac{\mathrm{df}(\mathrm{t})}{\mathrm{dt}}+\frac{\mathrm{d}^{1 / 2} \mathrm{f}(\mathrm{t})}{\mathrm{dt}^{1 / 2}}+\mathrm{f}(\mathrm{t})=\mathrm{u}(\mathrm{t})
$$

any initial conditions must specify how they are imposed. Specifically, a physical implementation of this system could contain three half-integrators, or two integrators and two half integrators. The initial condition specification must then also specify something structural. Also, this simple example clearly demonstrates that further study into the meaning of minimality for fractional order representations is necessary.

It should be apparent from this study that there is a clear connection between the fractional calculus and spatially distributed systems. Not only can spatially distributed system representations allow a better understanding of the fractional calculus, but the fractional calculus also allows a more compact representation and problem solution for some spatially distributed systems. 


\section{Appendix}

Equation (14) is repeated here for reference.

$$
\begin{aligned}
V(x, s) & =\frac{e^{+\sqrt{\frac{s}{\alpha}}}}{2}\left[V(0, s)+\frac{V^{*}(0, s)}{\sqrt{\frac{s}{\alpha}}}-\frac{1}{\alpha \sqrt{\frac{s}{\alpha}}} \int_{0}^{x} e^{-\sqrt{\frac{s}{\alpha}} \lambda} v(\lambda, 0) d \lambda\right] \\
& +\frac{e^{-\sqrt{\frac{s}{\alpha}}}}{2}\left[V(0, s)-\frac{V^{*}(0, s)}{\sqrt{\frac{s}{\alpha}}}+\frac{1}{\alpha \sqrt{\frac{s}{\alpha}}} \int_{0}^{x} e^{+\sqrt{\frac{s}{\alpha}} \lambda} v(\lambda, 0) d \lambda\right] .
\end{aligned}
$$

To get to Equation (16), which is the first term of Equation (14), set equal to zero, it is required to evaluate Equation (14) in the limit as X--> . In this limit, the second term in Equation (14) should go to zero due to the exponential, however, the integral inside the bracket will diverge. That is,

$$
\lim _{x \rightarrow \infty}\left(\frac{e^{-\sqrt{\frac{s}{\alpha}}} x}{2}\right)\left(\frac{1}{\alpha \sqrt{\frac{s}{\alpha}}} \int_{0}^{x} e^{+\sqrt{\frac{s}{\alpha}} \lambda} V(\lambda, 0) d \lambda\right)=0 \cdot \infty .
$$

We are thus left with an indeterminate form. This can be evaluated by using L'Hospital's rule and rearranging as follows

$$
\lim _{x \rightarrow \infty} \frac{\left(\frac{1}{2 \alpha \sqrt{\frac{s}{\alpha}}} \int_{0}^{x} e^{+\sqrt{\frac{s}{\alpha}} \lambda} V(\lambda, 0) d \lambda\right)}{\left(e^{+\sqrt{\frac{s}{\alpha}}} x\right)} .
$$

L'Hospital's rule says that this ratio has the same value as $t$ te ratio of the derivatives (with respect to $\mathrm{x}$ ) of the numerator and denominator. Differentiating the denominator is easy, but differentiating the numerator with respect to $\mathrm{x}$ requires the use of Leibniz $\mathrm{s}$ rule. Performing the differentiations gives

$$
\lim _{x \rightarrow \infty} \frac{\left(\frac{1}{2 \alpha \sqrt{\frac{s}{\alpha}}} \frac{d}{d x} \int_{0}^{x} e^{+\sqrt{\frac{s}{\alpha}} \lambda} V(\lambda, 0) d \lambda\right)}{\left(\sqrt{\frac{s}{\alpha}} e^{+\sqrt{\frac{s}{\alpha}}} x\right)} .
$$

Combining the leading constants, and applying the Leibniz's rule to the numerator, gives 


$$
\lim _{x \rightarrow \infty} \frac{\left(\frac{1}{2 s} e^{+\sqrt{\frac{s}{\alpha}}} x \mathrm{~V}(x, 0)\right)}{\left(e^{+\sqrt{\frac{s}{\alpha}}} x\right)}
$$

It can now be seen that the exponential terms cancel, which leaves the result

$$
\lim _{x \rightarrow \infty} \frac{1}{2 s} V(x, 0)=\frac{V(\infty, 0)}{2 s}
$$

The problem statement, however, requires that the boundary condition

$$
\mathrm{V}(\infty, \mathrm{t})=0
$$

be satisfied for all time. Thus it is shown that the first term of Equation (14) is equal to zero in the limit as $x \rightarrow \infty$. 


\section{References}

Bagley, R.L. and Calico, R.A., "Fractional Order State Equations for the Control of Viscoelastically Damped Structures," J. Guid., Cont., and Dyn., vol 14, no 2, pp 304-311, March-April 1991.

Bush, V., Operational Circuit Analysis, Wiley, 1929.

Carslaw, H.S. and Jeager, J.C., Operational Methods in Applied Mathematics. 2nd, Oxford, 1941.

Churchill, R.V., Operational Mathematics, McGraw-Hill, New York, 1958.

Courant, R. and Hilbert, D., Methods of Mathematical Physics, Interscience Publishers, New York, 1953.

Goldman, S., Transformation Calculus and Electrical Transients, Prentice-Hall, New York, 1949.

Heaviside, O., Electromagnetic Theory, Chelsea, New York, 1893/1971.

Holbrook, J.G., Laplace Transforms for Electronic Engineers, 2nd, Pergamon, Oxford, 1966.

Ichise, M., Nagayanagi, Y., and Kojima, T, "An Analog Simulation of Non-integer Order

Transfer Functions for Analysis of Electrode Processes," J. Electroanal. Chem. vol 33, pp 253-265, 1971.

Karplus, W.J., Analog Simulation: Solution of Field Problems, McGraw-Hill, New York, 1958.

Koeller, R.C., "Application of Fractional Calculus to the Theory of Viscoelasticity," J. Appl. Mech., vol 51, pp 299, June, 1984.

Koeller, R.C., "Polynomial Operators, Stieltjes Convolution, and Fractional Calculus in

Hereditary Mechanics," Acta Mechanica, vol 58, pp 251-264, 1986.

Lopez-Marcos, J.C., "A Difference Scheme for a Nonline ¿r Partial Integrodifferential Equation," SIAM J. Num. An., vol 27, no 1, pp 20-31, Feb., 1990.

Mandelbrot, B., "Some Noises with 1/f Spectrum, a Bridge Between Direct Current and White Noise," IEEE Trans. Inf. Thy., vol IT-13, no 2, April, 1967.

McBride, A.C., Fractional Calculus and Integral Transforms of Generalized Functions, Pitman, San Francisco, 1979.

McBride, A.C. and Roach, G.F., Editors, Fractional Calcı lus, Pitman, Boston, 1985.

Mikusinski, J., Operational Calculus, Pergamon, New York, 1959.

Oldham, K.B. and Spanier, J., The Fractional Calculus, Academic Press, San Diego, 1974.

Ross, B., Editor, Fractional Calculus and Its Applications. Springer-Verlag, Berlin, 1975.

Scott, E.J., Transform Calculus, Harper and Bros., 1955. 
Skaar, S.B., Michel, A.N., and Miller, R.K., "Stability of Viscoelastic Control Systems," IEEE Trans. Auto. Cont., vol 33, no 4, pp 348-357, April, 1988.

Starkey, B.J., Laplace Transforms for Electrical Engineers, Iliffe and Sons, London, 1954.

Sugimoto, N., "Burgers Equation with a Fractional Derivative; Hereditary Effects on Nonlinear Acoustic Waves," J. Fluid Mech., vol 225, pp 631-653, 1991.

Sun, H.H., Abdelwahab, A.A., and Onaral, B., "Linear Approximation of Transfer Function with a Pole of Fractional Order," IEEE Trans. Auto. Cont., vol AC-29, no 5, pp 441-444, May, 1984.

Sun, H.H., Onaral, B., and Tsao, Y., "Application of the Positive Reality Principle to Metal Electrode Linear Polarization Phenomena," IEEE Trans. Biomed. Eng., vol BME-31, no 10, pp 664-674, Oct. 1984. 


\section{REPORT DOCUMENTATION PAGE}

Public reporting burden for this collection of information is estimated to average 1 hour per response, includit g the time for reviewing instructions, searching existing data sources, gathering and maintaining the data needed, and completing and reviewing the collection of information. Send comments regarding this burden estimate or any other aspect of this Davis Highway, Surte 1204, Artington, VA $20202-4302$ and to burden, Davis Highway, Sure 1204, Artington, VA 22202-4302, and to the Office of Management and Budget, Paperwork Reduction Project (0704-0188). Washington, DC 20503.
1. AGENCY USE ONLY (Leave blank)
2. REPORT DATE
November 1998
3. REPORT TYPE AND DATES COVERED
Technical Memorandum

4. TITLE AND SUBTITLE

Insights Into the Fractional Order Initial Value Problem via Semi-infinite Systems

6. AUTHOR(S)

WU-523-22-13-00

Tom T. Hartley and Carl F. Lorenzo

7. PERFORMING ORGANIZATION MAME(S) AND ADDRESS(ES)

National Aeronautics and Space Administration

Lewis Research Center

Cleveland, Ohio 44135-3191

8. PERforming ORgANIZATION

REPORT NUMBER

E-11242

9. SPONSORING/MONITORING AGENCY NAME(S) AND ADDRESS(ES)

10. SPONSORINGMONITORING AGENCY REPORT NUMBER

National Aeronautics and Space Administration

Washington, DC 20546-0001

NASA TM-1998-208407

11. SUPPLEMENTARY NOTES

Tom T. Hartley, University of Akron, Akron, Ohio 44325-0001 and Carl F. Lorenzo, NASA Lewis Research Center. Responsible person, Carl F. Lorenzo, organization code 5500, (216) 43;-3733.

12a. DISTRIBUTIONVAVAILABILITY STATEMENT

Unclassified - Unlimited

Subject Categories: $59,66,31$, and 70

Distribution: Non:tandard

This publication is available from the NASA Center for AeroSpace Information, (301) 621 -0390

13. ABSTAACT (Max/mum 200 words)

This paper considers various aspects of the initial value problem for fractional order differential equations. The main contribution of this paper is to use the solutions to known spatially distributed systems to demonstrate that fractional differintegral operators require an initial condition term that is time-varying due to past distributed storage of information.

\section{SUBJECT TERMS}

Fractional calculus; Fractional order systems; Laplace transform; Diffusion equation; Lossy line; Initial value problem; Semi-infinite systems

\begin{tabular}{|c|c|c|}
\hline $\begin{array}{c}\text { 17. SECURITY CLASSIFICATION } \\
\text { OF REPORT } \\
\text { Unclassified }\end{array}$ & $\begin{array}{c}\text { 18. SECURITY CLASSIFICATION } \\
\text { OF THIS PAGE } \\
\text { Unclassified }\end{array}$ & $\begin{array}{c}\text { 19. SECURIT } \\
\text { OF CLASSIFICATION } \\
\text { OF ABST } \\
\text { UI classified }\end{array}$ \\
\hline
\end{tabular}

NSN 7540-01-280-5500 